\title{
Adaptation of Propagation Model Parameters toward Efficient Cellular Network Planning using Robust LAD Algorithm
}

\author{
Isabona Joseph ${ }^{\mathrm{a} *}$ and Divine O. Ojuh ${ }^{\mathrm{b}}$ \\ ${ }^{a}$ Department of Physics, Federal University Lokoja, Kogi State, Nigeria \\ ${ }^{\mathrm{b}}$ Department of Physical Sciences, Benson Idahosa University, Benin City, Nigeria
}

Received: 16 June 2020; Accepted: 20 July 2020; Published: 08 October 2020

\begin{abstract}
All new mobile radio communication systems undergo a cautious cellular network planning and re-planning process in order to resourcefully utilize the allotted frequency band and also ensure that the geographical area of focus is adequately fortified with integrated base stations transmitters. To this end, efficient radio propagation model prediction and tuning is of huge importance, as it assists radio network engineers to effectively assess and plan the cellular network signal coverage area. In this research work, an adaptive least absolute deviation approach is proposed and verified to fine-tune the parameters of Ericsson propagation model. The adaptive tuning technique have been verified experimentally with field propagation loss data acquired over three different suburban locations of a recently deployed LTE radio cellular network in Waterlines area of Port Harcourt City. In terms of the mean absolute percentage error and coefficient of efficiency, the outcomes of the proposed adaptive tuning approach show a higher degree of prediction performance accuracy on the measured loss data compared to the commonly applied least squares regression tuning technique.
\end{abstract}

Index Terms: Propagation loss, Adaptive propagation model tuning, Least square, Least absolute deviation.

\section{Introduction}

The growth of mobile telecommunication cellular systems and their networks have gone through noticeable progressive growth and development globally, ever since they were put into use or operation in the late 1980s. This growth is self-evident in the evolution, deployment and expansion of different cellular communication standards and infrastructures by telecommunication operators to cater for daily growth of mobile subscribers and enigmatic data demands. For sustenance of this growth, the mobile system cellular networks also need constant re-planning and adjustment. Signal coverage measurement, propagation loss analysis and predictions are distinctive aspects in the replanning of existing communication networks.

One fundamental aim of radio frequency (RF) coverage planning is to resourcefully utilize the allotted frequency band. As a result, RF coverage planning and prediction tools are of immense significance, as they assist radio network planers and designers to examine different system network configurations before and after deployment. However, the precision attained by the signal coverage prediction tool is also largely connected to the prediction accuracy of the radio propagation model applied [1,2]. For this reason, propagation model tuning with field measurement is a key component in the radio network coverage planning, design, deployment and management process. It is an activity in which realistic RF field measurements are conducted and employed to adapt the propagation model parameters in order to ensure accurate radio signal coverage predictions and propagation within intended planned network service area.

To adapt propagation model parameters, the analytic mathematical methods based on least-squares (LS) theory have mainly been the focused approach of most previous works in academic literatures (e.g. [3, 4, 5, 6, 7, 8, 9. 10, 11, 12]). For example, a LS based analytical approach is presented in [3], to tune the offset parameters of Hata model to effectively predict field measurement in Helsinki. Similar LS based analytical approaches are also adopted in [4, 5, 6, 7, 8]. In [9], a recursive algorithm based on LS theory is explored by the authors to calibrate the Okumura-Hata model for CMDA systems. The authors in [10], presented a linear LS method to fine-tune COST-231 model parameters for effective third generation network planning in Chinese City environment. In [11], a statistical LS method is explored to optimise the Hata model for GSM network planning in Baghdad City. In [12], a modified LS algorithm, which they tagged "Minimax LS algorithm" for the automatic tuning of Ericsson model is shown. An adaptive polynomial propagation model tuning based on LS algorithm is presented in [13]. 
The above commonly used LS method states that the requisite model parameters can obtained by minimizing the sum of squared error between the measured data and the predictions. A weighty problem arises with LS approach is that it usually performs poorly when breadth of outliers in the measured data is high. Particularly, The LS method also predicts poorly on highly none linear dataset $[14,15]$. Thus, a robust regression method to handle the above aforementioned problems is self-evident.

In this work, an Adaptive Least Absolute Deviation regression algorithm, which is tagged ALAD, is proposed to estimate and tune the parameters of Ericsson's propagation model for a robust prediction of measured propagation loss data. To validate superior performance of the proposed ALAD algorithm, its prediction performance will be examined using practical sample data based field measurement. The prediction performance of proposed ALAD algorithm will also be compared to the commonly used conventional LS approach.

The rest of the research work is presented as follows: a brief theoretical framework on Ericsson propagation model is presented in section 2. Material and methods adopted in the work which covers practical field measurement and parametric tuning of the Ericsson propagation model using the proposed adaptive least absolute deviation tuning algorithm with the field strength loss data is contained in section 3. Section 4 captures the results and discussion, while finally, a conclusion in drawn in section 5.

\section{Theoretical Framework}

\subsection{General Radio Propagation Modelling and Prediction Approaches}

As earlier mentioned, accurate estimation of radio wave signal strength and their losses from the transmitter to the receiver, is a vital step towards developing an effective cellular network planning and deployment. One of the fundamental drives targeted at getting abreast of the various elements that impact signal propagation loss is to enable one predict effectively the loss for a definite path, and as well estimate the coverage signal level of a broadcast station or base station.

When estimating the channel path losses either for plan a new radio system or optimizing an existing one, the first phase is to select the prediction path loss model for a particle environment. The second step is to adjust selected model via field measurement in order to accurately emulate and predict the characteristics of the environment. In general, the three key ways through which propagation loss modeling and predictions are made are outlined below:

- Statistical approach: This approach rely on measured signal data to predict propagation loss over cellular communication radio links. Examples of propagation models that were developed using this approach includes the Okumura Hata, Ericsson, Egli, Lee, COST-234 Hata, Ericsson etc. For example, COST-234 Hata, model was formulated by EUROCOST based on field propagation measurements conducted by Okumura and Hata in Japan.

- Deterministic approach: This approach employs the fundamental laws physics for radio propagation loss calculation and prediction. In terms of accuracy, this approach loss prediction preferred to the statistical approach. Databases of topography elevations, clutter heights and atmospheric refractivity settings are all applied in the deterministic prediction process. The Free space, ray tracing and plain earth model are key examples of deterministic propagation prediction modelling approach.

- Semi-Deterministic Approach: This approach combines deterministic and empirical prediction approach for propagation loss prediction. Typical examples of predictive modelling formed using semi-deterministic approach are Walfisch-Bertoni and Walfisch-Ikegami models. For example, the Walfisch-Ikegami was formulated based combined efforts of Ikegami and Walfisch-Bertoni for propagation loss calculations and prediction of base station transmitters positioned above the mean building roof height level.

\subsection{Ericsson Propagation Model}

For effective radio-propagation calculation and predictions of transmitted signal attenuation loss over the transmitter-receiver communication channels of cellular system networks, the empirically based Ericsson model, also known as Algorithm 9999, is one of the commonly used models. Specifically, this model has been integrated into Telephone Mobile Software (TEMS) planning tool [16]. The Ericsson model is built on modified Okumura-Hata to provide means of adjusting of its parameters for propagation environment adaptation. Base on the TEMS planning tool, Ericsson model is given as $[12,17]$ :

$$
P L_{\text {Eric }}=P_{0}+P_{1} \log r+P_{2} \log H_{\text {eff }}+P_{3} \log r \cdot \log H_{e f f}-3.2\left[\log \left(11.75 H_{m}\right]^{2}+w(f)\right.
$$

Where

$$
w(f)=44.49 \log f-4.78(\log f)^{2}
$$


With $H_{e_{f} f}$ and $r$ being the effective base station height and its distance to mobile station. The constants $P_{o}$, $P_{1}, P_{2}$, and $P_{3}$ are distinctive model tuning parameters and their default values are given in table 1.

Table 1. Ericsson model Parameters for different Environment [12, 17].

\begin{tabular}{|c|c|c|c|}
\hline Parameter & Rural & Suburban & Urban \\
\hline Po & 45.95 & 43.2 & 36.2 \\
\hline P1 & 100.6 & 68.93 & 30.2 \\
\hline P2 & 12 & 12 & 12 \\
\hline P3 & 0.1 & 0.1 & 0.1 \\
\hline
\end{tabular}

\section{Materials and Methods}

This section provides the methodology employed to actualize the proposed ALAD regression algorithm approach. The ALAD iteratively uses weights to minimize the sum absolute error of the residuals as described using equations (5) to (11) and section 3.2. In the procedure, a detailed field strength data measurement is first conducted to compute and analyze path attenuation loss characteristics of transmitted signals at different locations of the study area, which is a typical urban terrain. Secondly, with the aid of Matlab 2018a software optimisation and computation environment, combined with the measured computed path loss data, the proposed ALAD solution approach is employed to adaptively fine-tune the urban parameters of the Ericsson model as displayed in Table 1. This is with a goal to minimalize the sum of the absolute squares variances amid the measured loss and the original Ericsson model. Finally, first order statistics metrics like mean prediction error (MPE) and root mean square error (RMSE) are used to evaluate the effectiveness our proposed ALAD path loss model tuning approach in comparison with other commonly used LS technique.

\subsection{Field Measurement}

With the capability of TEMS integrated drive test equipment, housed in a gulf car, field strength data expressive of Reference Signal Received Power (RSRP), were acquired from three operational LTE cellular base station sites in Waterlines areas of Port Harcourt city. Each of the study cell site is empowered with three sectored directional antennas. The cell sites locations were carefully chosen to reflect a good blend of various commercial and uneven residential structures that are permeate the study area. Thus, the area provides a distinctive terrain for measurement study of propagated LTE radio signals at 2600MHz. Sincerely, to our best knowledge with regard to academic literature, this is the first time this type of research work is being embarked upon in the study area.

The field signal measurement was conducted up to a distance of $1500 \mathrm{~m}$ for site $1,1300 \mathrm{~m}$ for site 2 and $560 \mathrm{~m}$ for site 3; the measured signal data consists of over 2000 data points, which are average to 600 readings. Shown in Fig. 1 is the snap shot of the drive test routes with TEMS tools in location. In this figure, the red, yellow and green colours indicate bad, fair and good RSRP qualities, respectively, along the drive test routes. The snap shot clearly reveals that a larger portion of the drive test routes is red, thus indicating poor RSRP quality in study location. One of the key factors that can lead to such a high poor signal coverage level quality in terms of RSCP values is improper network planning. Hence, the need for optimised propagation loss model prediction for improve network planning in the study location as proposed in this work is self-evident.

To ensure that the influence of small-scale fading on the propagation is catered for, all measured RSRP values were further post-processed to a single mean value, along the test routes and locations. The field test locations were kept track of using the GPS unit. The propagation loss, PLi, (dB) values have been obtained from the RSRP by:

$$
P L_{\text {mea }, i}=\operatorname{EIRP}+G_{A, i}-R_{\text {meas }, i}
$$

where EIRP and $G_{A}$ are special antenna resource parameters.

The path loss and RSRP are related to electric field strength, $E(d B \mu V / m)$ by $[18,19,20]$ :

$$
E(d B \mu V / m)=E I R P-P L_{\text {mea }}, i+20 \log f+109.35
$$




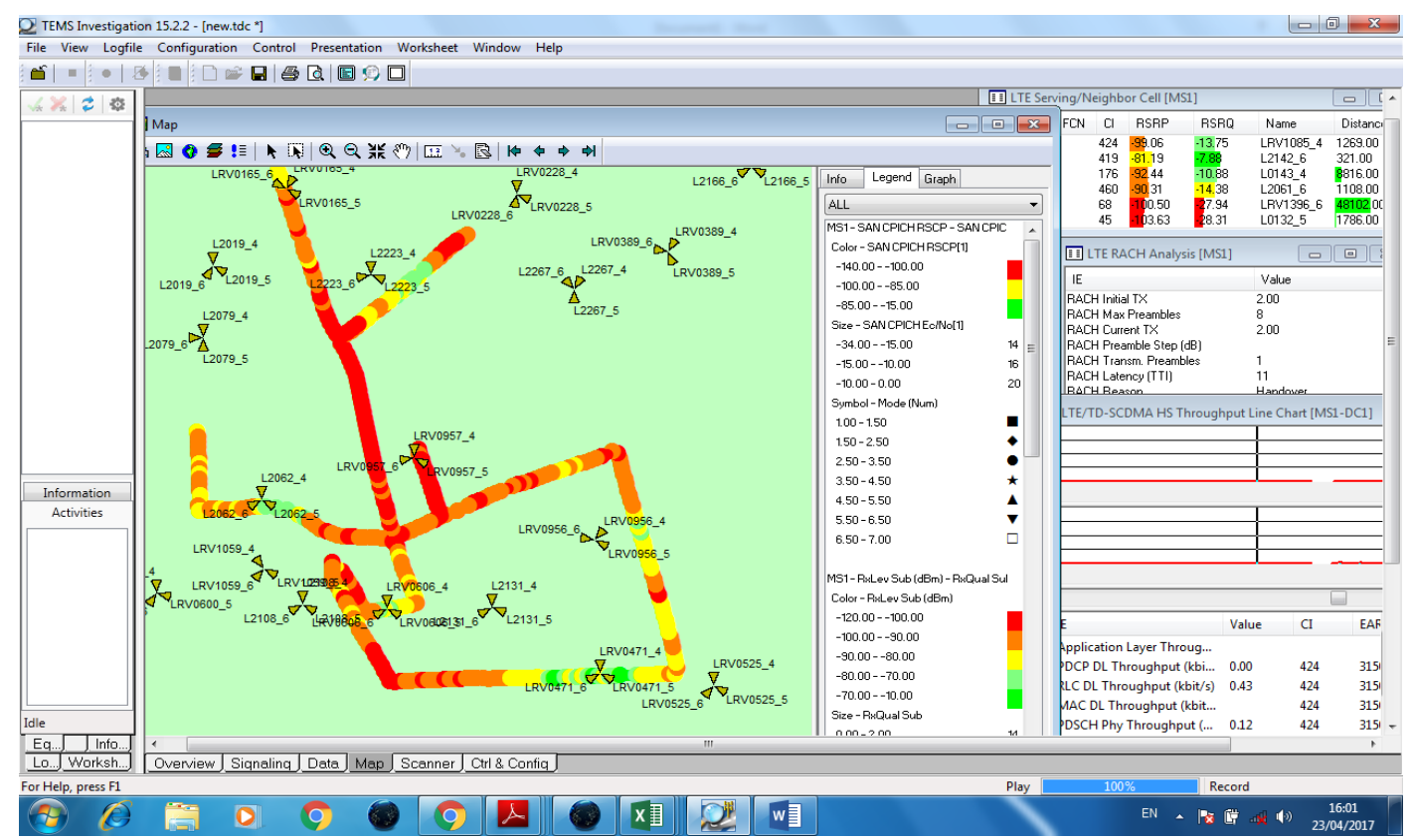

Fig. 1. A snap shot of the drive test routes of the acquired propagated RSRP data in study location

\subsection{Parameter Tuning of Ericsson Model with ALAD Algorithm}

The LAD adaptation solution approach entails adjusting the coefficients of the Ericsson model with a goal to minimalize the sum of the absolute squares variances amid the measured loss and simulations.

To apply the proposed ALAD algorithm, we start by streamlining the Ericsson model of equation (1) to give:

$$
P L_{\text {Eric }}=P_{0}+P_{1} \log r+Q
$$

where

$$
Q=P_{2} \log H_{e f f}+P_{3} \log r \cdot \log H_{e f f}-3.2\left[\log \left(11.75 H_{m}\right]^{22}+w(f)\right.
$$

Furthermore, for $X=\log r, A=P_{0}+Q$ and $b=P_{1}$, equation (5) becomes

$$
P L_{\text {Eric }}=A+B X
$$

Let deviations the measured propagation loss and loss prediction attained using the Ericsson model be denoted by:

$$
\left.E(A, B)=\sum \mid P L_{\text {meas }, i}-A X_{i}-B\right) \mid
$$

where $E(\beta, b)$ represent the deviation, $P L_{\text {meas }, i}$, is the measured loss and i stands for the observation.

By means of the ALAD regression approach, the parameters A and B are can be found by minimising the objective function:

$$
\left.\min E(A, B)=\sum \mid P L_{\text {meas }, i}-A X_{i}-B\right) \mid
$$

Subject to $E(A, B)=P L_{\text {mea }}-A X-B$ for all i

$0 \geq E(A, B) \leq 0,0 \leq A, B \leq 0$ for all

Now, if we fix the value of $A$, say $A=A_{0}$, the cost function (8) turn into a sole-parameter function of $B$ :

$$
E(B)=\sum_{i}^{K}\left|P L_{\text {mea }, i}-A_{o} X_{i}-B\right|
$$

And the parameter B can be determined as the weighted median: 


$$
B=\operatorname{MED}\left(P L_{\text {mea }, i}-\left.A_{o} X_{i}\right|_{i=1} ^{K}\right)
$$

Also, If we fix the value of $\mathrm{B}$, i.e. $\mathrm{B}=\mathrm{B}_{\mathrm{o}}$, the cost function (8) leads to:

$$
\begin{gathered}
E(A)=\sum_{i=1}^{K}\left|P L_{\text {mea }, i}-A_{o} X_{i}-B\right| \\
=\sum_{i}^{K}\left|X_{i}\right|\left|\frac{P L_{\text {meas }},{ }_{i}-B_{o_{i}}}{X_{i}}-A\right|
\end{gathered}
$$

And the parameter A can be calculated as the weighted median:

$$
A=\operatorname{MED}\left(\left.\left|X_{i}\right| \diamond \frac{P L_{\text {mea }, i}-B_{o}}{X_{i}}\right|_{i=1} ^{K}\right)
$$

where $\diamond$ expresses the replication operator.

The proposed optimal Ericsson model tuning approach using ALAD is summarised below in algorithm 1.

Algorithm 1: Optimal Tuning of Ericsson Model using ALAD

1) Inputs: $A, B, X, P L_{\text {meas }, i}$;

2) Set $X_{i}=-(K+1)$ if $K$ is even, set $X_{i}=-\frac{(K+1)}{2}+i$ if $K$ is odd;

3) choose $0 \leq A, B \leq 0,0 \geq E(A, B) \leq 0$, for all i ;

4) set $E(A, B)_{\min }=\infty$;

5) while $0 \geq E(A, B)_{\min } \leq 0$ do;

6) for all $X_{\mathrm{i}}$ do;

7) Calculate $B=\operatorname{MED}\left(P L_{\text {mea }, i}-\left.A_{o} X_{i}\right|_{i=1} ^{K}\right)$ for fix the value of $\mathrm{A}$

8) Calculate $A=\operatorname{MED}\left(\left.\left|X_{i}\right| \diamond \frac{P L_{\text {mea }, i}-B_{o}}{X_{i}}\right|_{i=1} ^{K}\right)$ for fix the value of B;

9) Calculate $\left.E(A, B)=\sum \mid P L_{\text {meas }, i}-A X_{i}-B\right) \mid$

19) if $E(A, B)=E(A, B)_{\min }$ then

11) $E(A, B)_{\min }=E(A, B)$

12) end while

13) end if

14) end for

15) return $E(A, B)_{\min }, A_{\text {best }}, B_{\text {best }}$

\subsection{Performance Measures}

The lesser the deviancy between the measures and the resultant prediction, the more appropriate is the prediction process. In order to measure the amount of this deviation, the first-order based statistical metrics have been used. The statistical metrics employed were mean prediction error (MPE), and root mean square error (RMSE), each computed in the order of equations (14) and (15). The larger the values of MPE, MAPE and RMSE are, the disproportionate and poorer the prediction.

The correlation coefficient (COC) and coefficient of efficiency (COE), expressed in equations (16) and (17) were two other important statistical performance metrics utilised for evaluation. Both metrics quantify and reveal precisely how well the predicted propagation loss model tracks the measured loss. The nearer the values of COC and COE are to one, the greater and better the correlation between the measures and the resultant prediction.

$$
\text { RMSE }=\frac{1}{N} \sqrt{\sum_{q=1}^{N}\left[y_{q}-d_{q}\right]^{2}}
$$




$$
\begin{gathered}
C O C=\frac{\sum_{q=1}^{N}\left(y_{q}-\bar{y}_{k}\right)\left(y_{q}-\bar{d}_{k}\right)}{\sqrt{\left[\sum_{q=1}^{N}\left[\left(y_{k}-\bar{y}_{k}\right)^{2}\right]\right]\left[\sum_{q=1}^{N}\left[\left(y_{k}-\bar{d}_{k}\right)^{2}\right]\right]}} \\
C O E=1-\frac{\sum_{q=1}^{N}\left[y_{q}-d_{k}\right]^{2}}{\sum_{k=1}^{\text {Kest }}\left[y_{q}-\bar{y}_{k}\right]^{2}}
\end{gathered}
$$

where $y_{q}$ denotes the desired target output, $d_{q}$ indicates the actual network output, $\bar{y}_{q}$ is the mean of the actual network output, $q=1,2, \ldots, N$ are values the signal power

\section{Results and Analysis}

The graphs in Figs. 2(a), 3(a) and 4(a) are provided to reveal how the Ericson model predict the measured loss data in sites 1 to 3; and from the graphs, it is noticeably seen that the Ericsson model path loss over predicted the measured loss values by a difference of 4 to $20 \mathrm{~dB}$ in site 1,5 to $40 \mathrm{~dB}$ in site 2 and 3 to $25 \mathrm{~dB}$ in site 3 . Among other things, the poor prediction made by the Ericsson model can ascribe to several reasons. One of it is that the physical terrain of Nigeria is to a certain extent is different from the environment where the empirical-based Ericsson model is developed. Secondly, the differences in terrestrial and topological condition of Nigeria over where Ericson model is developed are another reason for its poor prediction.

Next is to evaluate the effectiveness of our proposed LAD solution approach employed to adaptively fine-tune the urban parameters of the Ericsson model path loss in comparison with LS-based conventional approach. The plots in Figs. 2(b) and 2(c), 3(b) and 3(c) and 4(b) and 4(c) shows the respective adapted Ericsson model to the measured loss data using LS-based approach and LAD approach. The scatter plotted graphs in Figs. analyses the mean prediction error realised using the original Ericson model and the prediction error made after its adaptation by employing LS-based approach and LAD approach. The prediction indicated by a red line in Figs. 2(b) and 2(b), 3(b) and 3 (b), 4(c) and 5(c) minimizes the LS and LAD of the error. The Ericson model parameters before and after adaptation are provided in Table 2. A larger the prediction error distribution along the loss data test points, the poorer the Ericsson model made before its adaptation and after adaptation. The degree of prediction accuracy attained in terms of RMSE, R and COE on the measured loss data after the adaptation of Ericson model with LS and LAD-based approach are displayed in table 3 for the respective study sites. The results disclosed that the LAD-based Ericson model adaptation approach outperform the LS-based Ericson adaptation approach in the three study location. For example, with RMSE performance assessment indicators, LAD-based Ericson achieved $3 \mathrm{~dB}$ for site $1,4 \mathrm{~dB}$ for site 2 and $3.5 \mathrm{~dB}$ for site 3 , compared to the LS-based Ericson which achieved a higher prediction error values of $5 \mathrm{~dB}$, and $7 \mathrm{~dB}$ and $06.5 \mathrm{~dB}$ for site 1 to 3 , respectively.
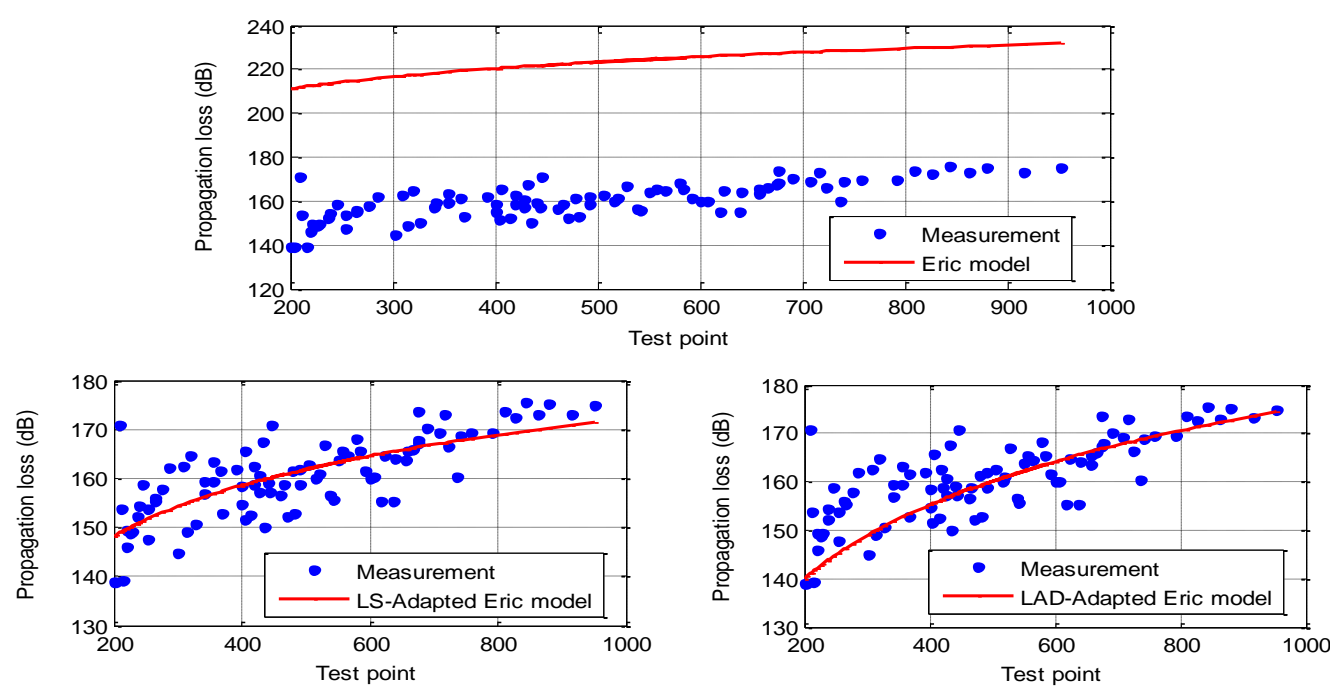

Fig. 2. Propagation loss prediction along field test points using Ericsson model, LS-Adapted Ericsson mode and LAD-Adapted Ericsson mode in location 

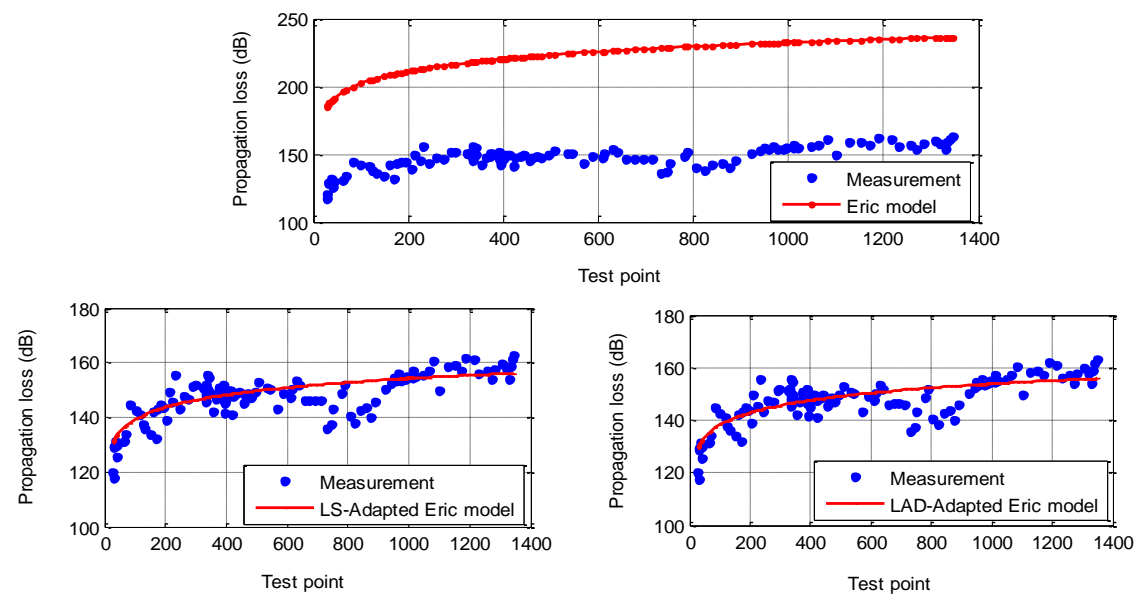

Fig. 3. Propagation loss prediction along field test points using Ericsson model, LS-Adapted Ericsson mode and LAD-Adapted Ericsson mode in location 2.
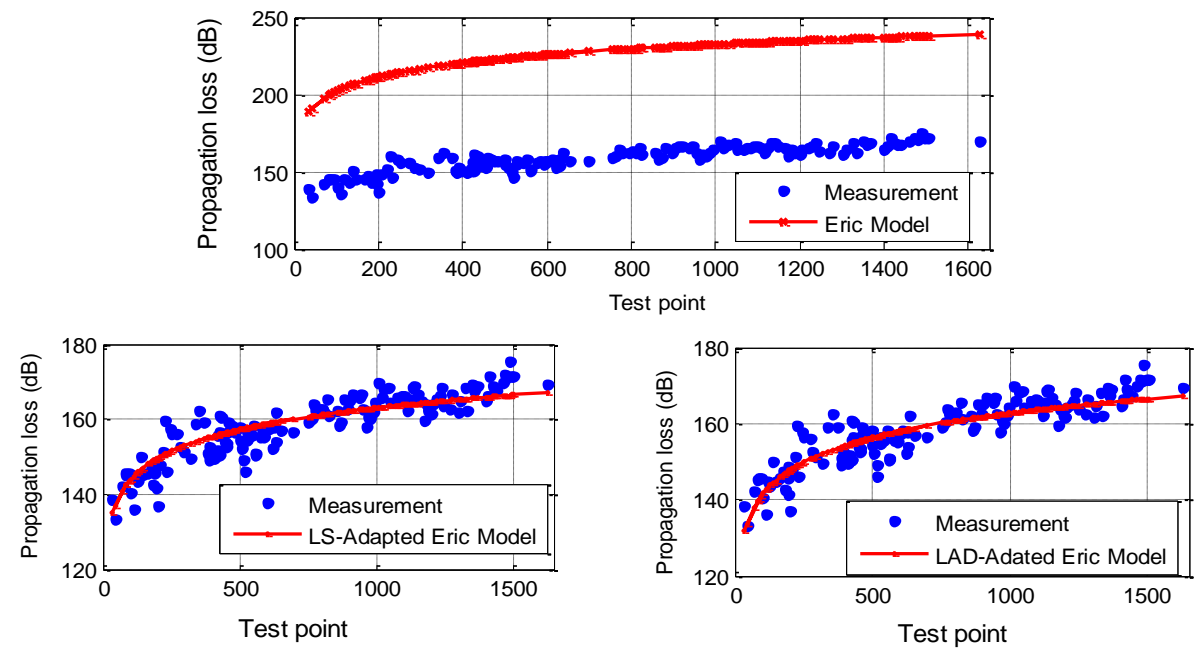

Fig. 4. Propagation loss prediction along field test points using Ericsson model, LS-Adapted Ericsson mode and LAD-Adapted Ericsson mode in location 3.
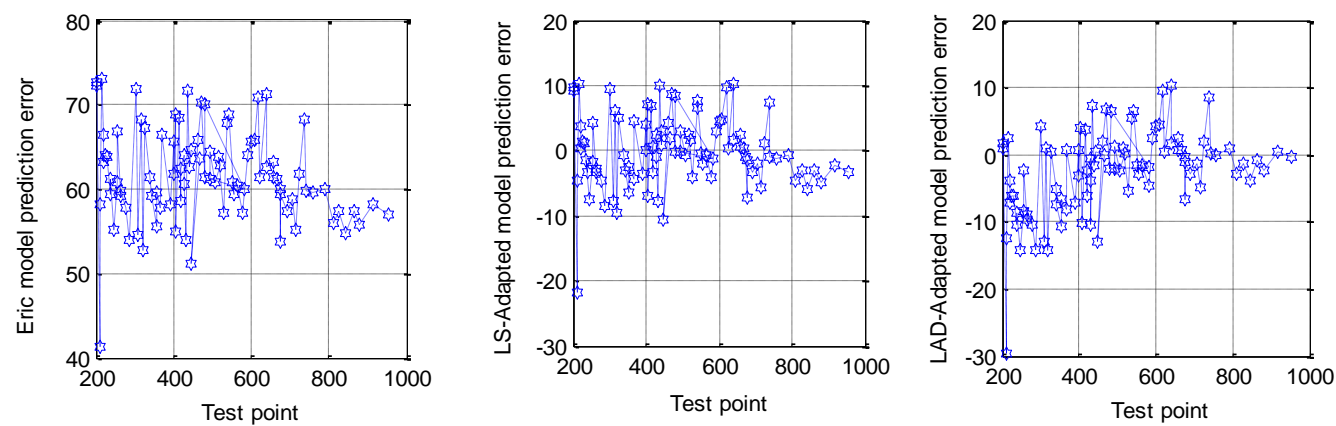

Fig. 5. Mean propagation loss prediction error along field test points using Ericsson model, LS-Adapted Ericsson mode and LAD-Adapted Ericsson mode in location 1 . 

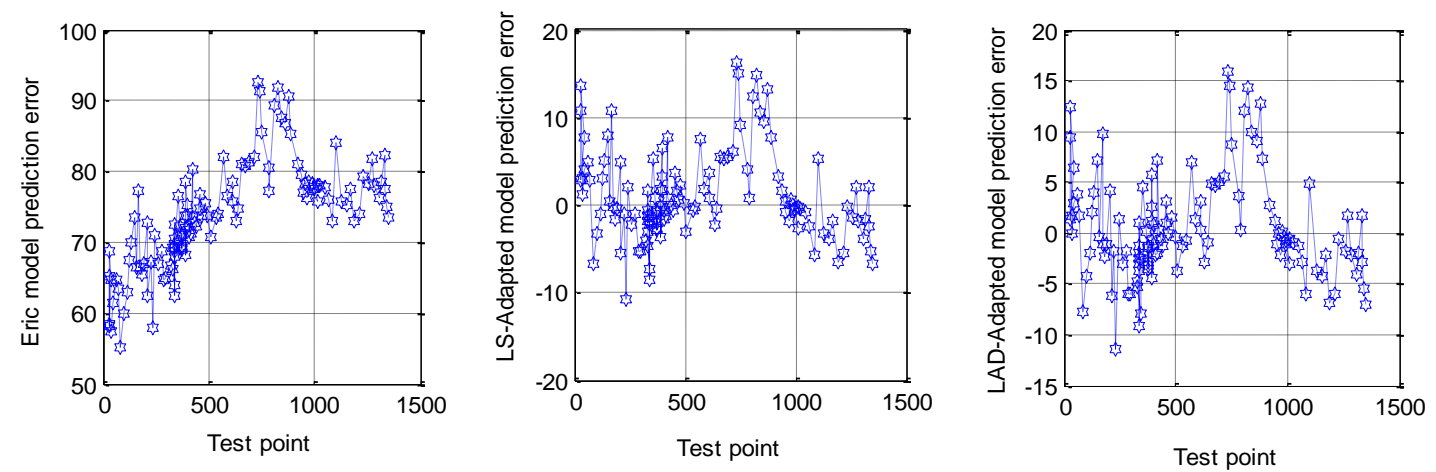

Fig. 6. Mean propagation loss prediction error along field test points using Ericsson model, LS-Adapted Ericsson mode and LAD-Adapted Ericsson mode in location 2 .
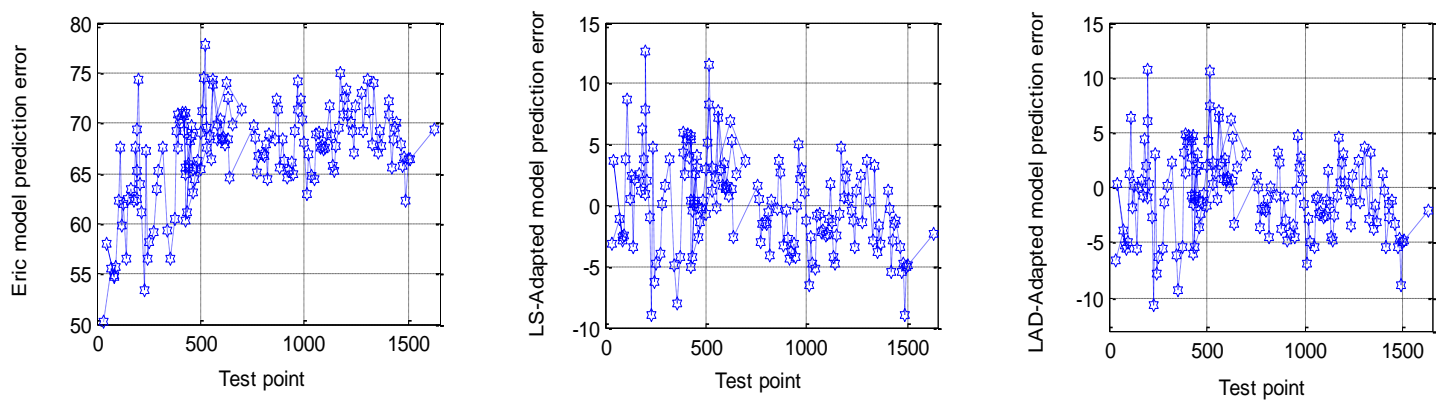

Fig. 7. Mean propagation loss prediction error along field test points using Ericsson model, LS-Adapted Ericsson mode and LAD-Adapted Ericsson mode in location 3.

The prediction performances LS and LAD model parameter tuning using R and COE metrics, presented in Fig. 8 to 10 . Both metrics quantify and reveal precisely how well the predicted propagation loss model tracks the measured loss. The nearer the values of $\mathrm{COC}$ and $\mathrm{COE}$ are to one, the greater and better the correlation between the measures and the resultant prediction. The results summary in Table 3 disclose the proposed LAD model tuning approach achieve COE of 5, 4, 10\% in location 1, COE of 5, 4, 10\% in location 2 and COE of 5, 4, and $10 \%$ in location 3 over the standard LS approach respectively. The same better prediction accuracy is also achieved with $R$ values in using the LAD adaptation approach.
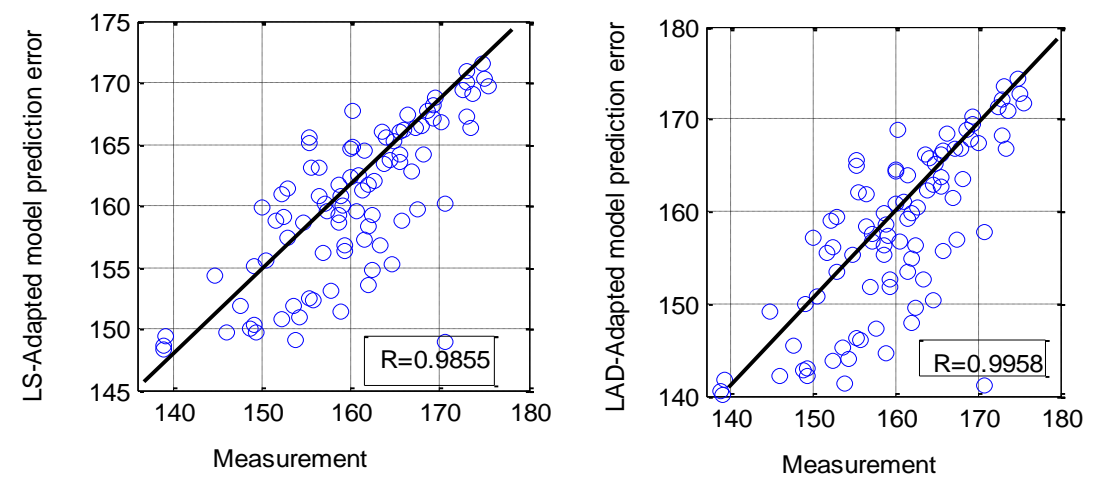

Fig. 8. A plot of Predicted loss data versus field measurement using LS-Adapted Ericsson model and LAD-Adapted Ericsson model in location 1. 

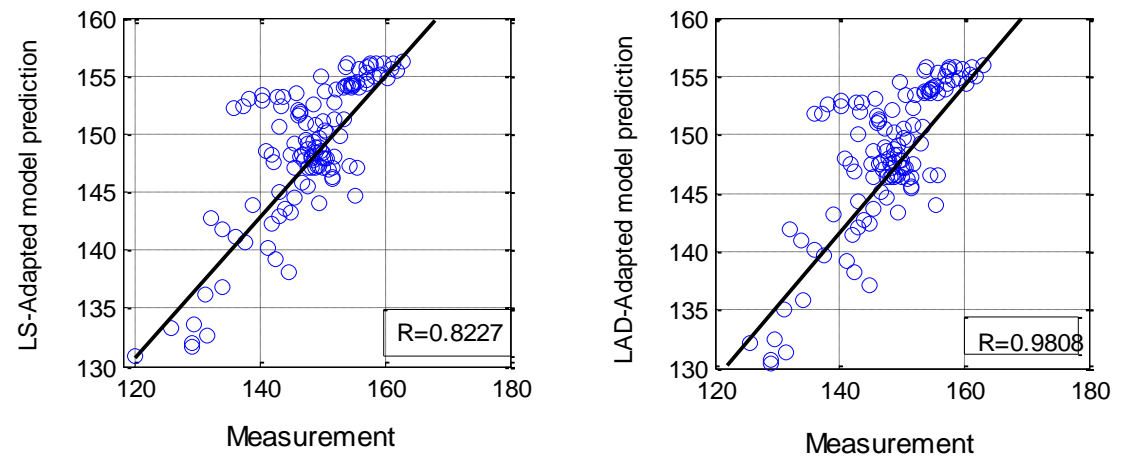

Fig. 9. A plot of Predicted loss data versus field measurement using LS-Adapted Ericsson model and LAD-Adapted Ericsson model in location 2.
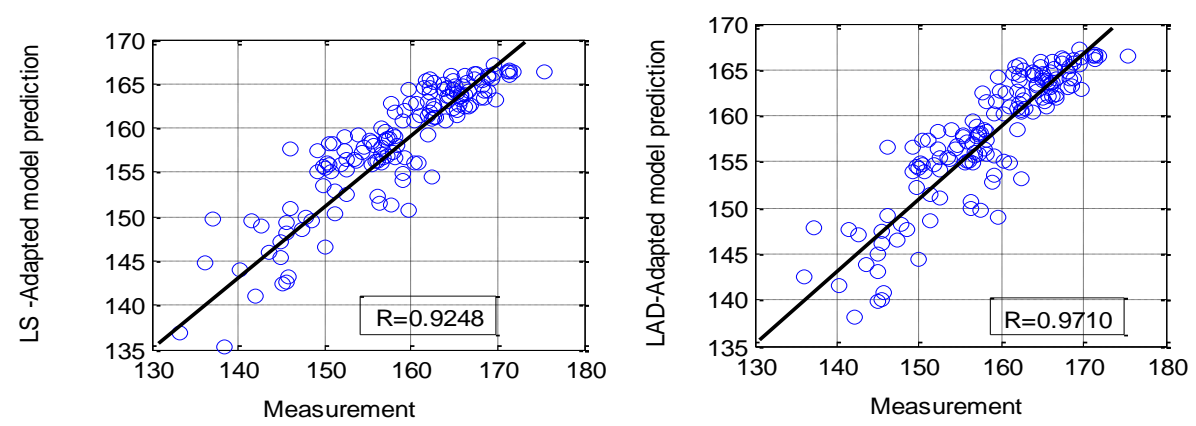

Fig. 10. A plot of Predicted loss data versus field measurement using LS-Adapted Ericsson model and LAD-Adapted Ericsson model in location 3.

Table 2. Ericsson model parameters before and after tuning with Adapted LS and LAD Algorithm in locations 1 -3.

\begin{tabular}{|c|c|c|c|c|}
\hline Location & $\begin{array}{l}\text { Default Ericsson } \\
\text { model Parameters }\end{array}$ & $\begin{array}{l}\text { Model Parameters } \\
\text { Before Tuning }\end{array}$ & $\begin{array}{l}\text { Tuned Model } \\
\text { Parameters with LS } \\
\text { approach }\end{array}$ & $\begin{array}{l}\text { Tuned Model } \\
\text { Parameters with } \\
\text { LAD approach }\end{array}$ \\
\hline \multirow{4}{*}{1} & A & 36.2 & 0.10 & 19.10 \\
\hline & B & 30.2 & 6.93 & 19.90 \\
\hline & C & 12 & \multirow{2}{*}{\multicolumn{2}{|c|}{$\begin{array}{l}12 \\
0.1\end{array}$}} \\
\hline & D & 0.1 & & \\
\hline \multirow{4}{*}{2} & A & 36.2 & 3.87 & 14.90 \\
\hline & B & 30.2 & 1.64 & 15.51 \\
\hline & $\bar{C}$ & 12 & \multirow{2}{*}{\multicolumn{2}{|c|}{0.1}} \\
\hline & D & 0.1 & & \\
\hline \multirow[t]{4}{*}{3} & A & 36.2 & -35.85 & 34.13 \\
\hline & B & 30.2 & -6.87 & 21.05 \\
\hline & C & 12 & \multirow{2}{*}{\multicolumn{2}{|c|}{$0.1^{12}$}} \\
\hline & $\mathrm{D}$ & 0.1 & & \\
\hline
\end{tabular}

Table 3. Accuracy Evaluation Results using LS-Adapted Ericsson model and LAD-Adapted Ericsson model in locations 1 to 3.

\begin{tabular}{|c|c|c|c|c|}
\hline Location & Reg & RMSE & $\mathrm{R}$ & $\mathrm{CoE}$ \\
\hline \multirow{2}{*}{1} & LS-Adapted Ericsson Model & 3.46 & 0.9248 & 0.8553 \\
\hline & LAD-Adapted Ericsson Model & 2.45 & 0.9668 & 0.9347 \\
\hline \multirow[t]{2}{*}{2} & LS-Adapted Ericsson Model & 5.05 & 0.8227 & 0.6443 \\
\hline & LAD-Adapted Ericsson Model & 2.75 & 0.9808 & 0.9620 \\
\hline \multirow{3}{*}{3} & & & & \\
\hline & LS-Adapted Ericsson Model & 7.64 & 0.9817 & 0.9639 \\
\hline & LAD -Adapted Ericsson Model & 2.47 & 0.9958 & 0.9917 \\
\hline
\end{tabular}


To further illustrate the positive effect from the superior prediction accuracy of the proposed ALAD model tuning algorithm in the perspective network planning, we have considered the electric field strength parameter, obtained from the predicted propagation loss values using the expression in equation (5). Figs. 11 to 13 compares the field strength distributions at three study locations, and percentile values are shown in Table 4 for the ALAD and LS algorithms. According to Figs. 5 to 7 and table 4, the ALAD algorithm yield gains of 7, 10 and 15\% in location 1, a gain of 5, 4 and $10 \%$ in location 2 and a gain of 6,5 , and $9 \%$ in location 3 over the standard LS approach respectively, at 25, 50 and 75 cumulative distribution percentiles. The superior prediction accuracy results with proposed ALAD algorithms clearly indicates that it yield a better field strength coverage area prediction around the study locations when employed in RF cellular coverage planning.

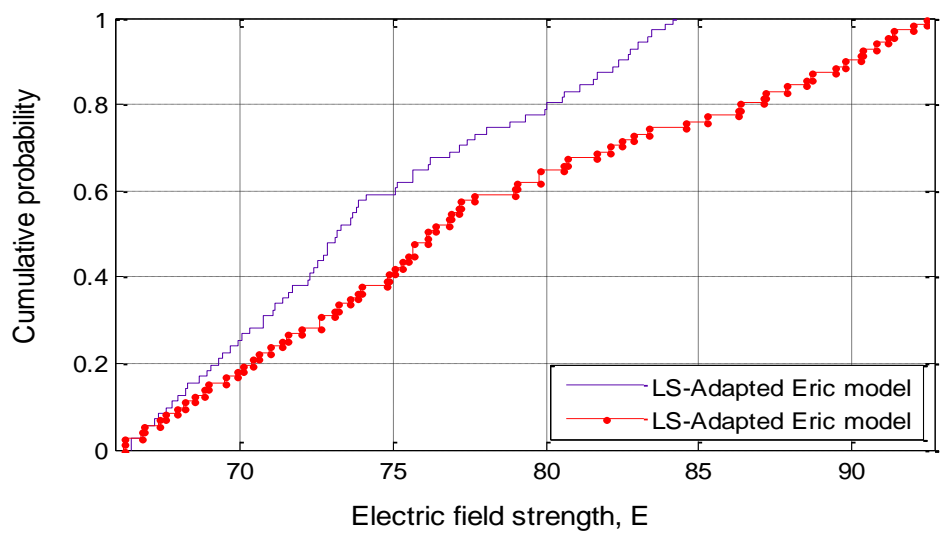

Fig. 11. CDF plot of Predicted Electric field strength data using LS-Adapted Ericsson model and LAD-Adapted Ericsson model in location 1.

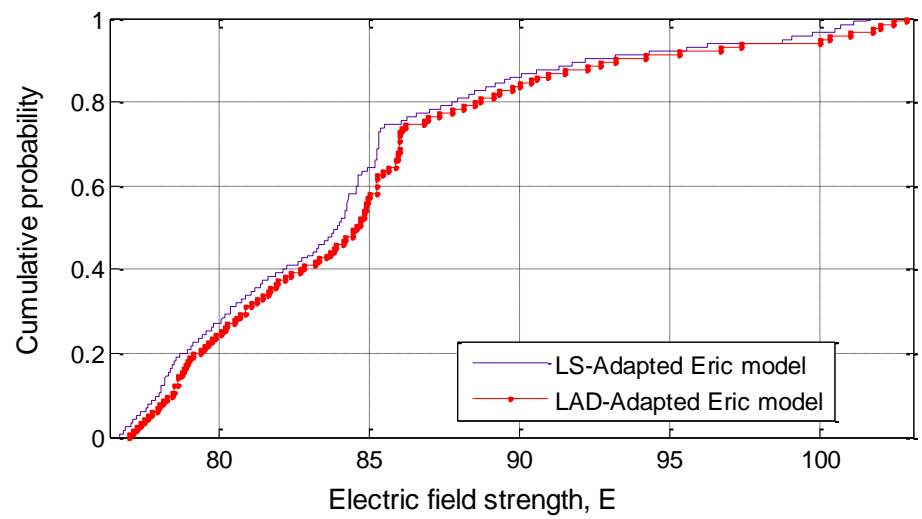

Fig. 12. CDF plot of Predicted Electric field strength data using LS-Adapted Ericsson model and LAD-Adapted Ericsson model in location 2.

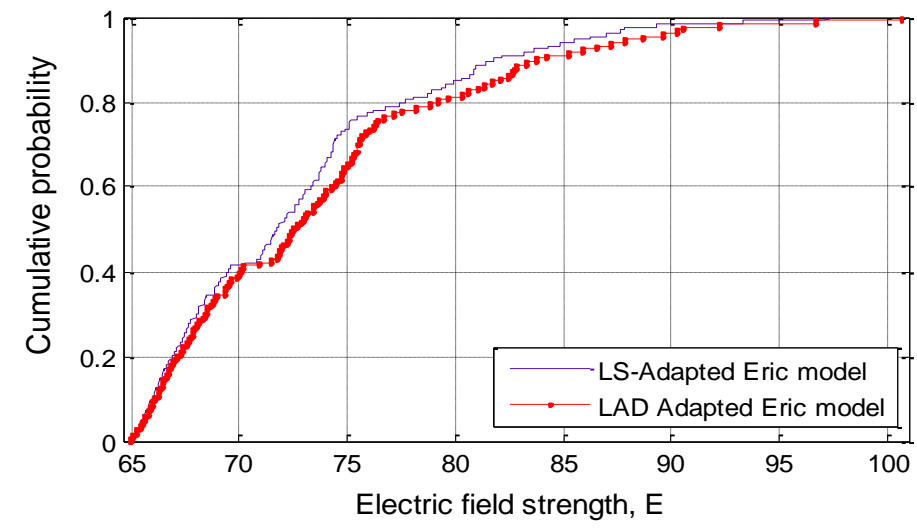

Fig. 13. CDF plot of Predicted Electric field strength data using LS-Adapted Ericsson model and LAD-Adapted Ericsson model in location 3. 
Table 4. Predicted Electric Field strength (E) at 25, 50 and 75 percentile using LS-Adapted Ericsson model and LAD

\begin{tabular}{|c|c|c|c|}
\hline & $\mathrm{E}^{25}(\mathrm{~dB} \mu \mathrm{V} / \mathrm{m})$ & $\mathrm{E}^{50}(\mathrm{~dB} \mu \mathrm{V} / \mathrm{m})$ & $\mathrm{E}^{75}(\mathrm{~dB} \mu \mathrm{V} / \mathrm{m})$ \\
\hline LS-Adapted Ericsson Model & 69.20 & 73.55 & 77.82 \\
\hline LAD-Adapted Ericsson Model & 73.36 & 77.44 & 84.49 \\
\hline LS-Adapted Ericsson Model & 81.66 & 83.76 & 86.89 \\
\hline LAD-Adapted Ericsson Model & 83.45 & 85.01 & 75.00 \\
\hline LS-Adapted Ericsson Model & 67.21 & 72.11 & 77.16 \\
\hline LAD-Adapted Ericsson Model & 68.33 & 74.01 & \\
\hline
\end{tabular}

Adapted Ericsson model in location 3.

\section{Conclusion}

An Adaptive least absolute deviation approach was proposed and illustrated in this research to fine-tune the parameters of Ericsson propagation model. The adaptive tuning technique was experimentally verified with field propagation loss data acquired over three different suburban locations of a recently deployed LTE radio cellular network in Port Harcourt, Nigeria. In terms of the mean absolute percentage error and coefficient of efficiency, the outcomes of the proposed adaptive tuning approach show a high degree of prediction performance improvement on measured loss data over commonly applied least-squares regression method. Also, during results validation, the ALAD algorithm yielded gains of 7, 10 and 15\% coverage level estimation performance in location 1, gains of 5, 4 and 10\% in location 2 and gains of 6,5 , and $9 \%$ in location 3 over the standard LS approach respectively, at 25, 50 and 75 cumulative distribution percentiles. These superior prediction accuracy results with proposed ALAD algorithms clearly indicates that it yield a better field strength coverage area prediction around the study locations when employed in RF cellular coverage planning.

In future research work, we intent to explore other propagation models adaptation techniques such as the iterative least regression and global optimisation algorithms using urban, suburban and rural areas as case studies.

\section{References}

[1] Isabona J., Srivastava V. M. Coverage and Link Quality Trends in Suburban Mobile Broadband HSPA Network Environments. Wireless personal Communications, 95, 3955-3968, 2017.

[2] Mardeni R., Lee Y. P. Y. P. The Optimization of Okumura's Model for Code Division Multiple Access (CDMA) System in Malaysia. European Journal of Scientific Research, 45 (4), 508-528, 2010.

[3] AarnæS E., Holm S. Tuning of empirical radio propagation models effect of location accuracy. Wireless Personal Communications, 30 (2-4), 267-281, 2004.

[4] Chimaobi N.N., Nnadi C.C., Nzegwu A.J. Comparative Study of Least Square Methods for Tuning Erceg Pathloss Model. American Journal of Software Engineering and Applications. 6 (3), 61-66, 2017.

[5] Isabona J., Konyeha C.C. Urban Area Path loss Propagation Prediction and Optimisation Using Hata Model at 800MHz. IOSR Journal of Applied Physics (IOSR-JAP), 3 (4), 8-18, 2013.

[6] Castro B. S., Pinheiro M. R., Cavalcante G. P., Gomes I. R., Carneiro O. d. O. Comparison between known propagation models using least squares tuning algorithm on $5.8 \mathrm{GHz}$ in Amazon region cities. Journal of Microwaves, Optoelectronics and Electromagnetic Applications, 10 (1), 106-113, 2011.

[7] Isabona J., Azi S. Optimised Walficsh-Bertoni Model for Pathloss Prediction in Urban Propagation Environment. International Journal of Engineering and Innovative Technology (IJEIT), 2 (5), 14-20, 2012.

[8] Castro-Hernandez D., Paranjape R. Local Tuning of a Site-Specific Propagation Path Loss Model for Microcell Environments, International Journal of Wireless Personal communications, 91 (2), 709-728, 2016.

[9] Liming X., Dacheng Y. A Recursive Algorithm for Radio Propagation Model Calibration based on CDMA Forward Pilot Channel, In Proceedings of IEEE $14^{\text {th }}$ International Symposium on Personal, Indoor and Mobile Radio Communication, 970972, 2003.

[10] Mingjing Yang., Wenxiao Shi. A linear least square method of propagation model tuning for 3G radio network planning. In Fourth International Conference on Natural Computation, 5150-154, 2008.

[11] Mohammed A.K., Jaafar A.A. Performance Evaluation of Path Loss in Mobile Channel for Karada Distric in Baghdad City. Engineering and Technical Journal, 30 (17), 3023-3038, 2012.

[12] Simic I. L., Stanic I., Zrnic B. Minimax LS algorithm for automatic propagation model tuning, Proceeding of the 9th Telecommunications Forum, Belgrade, Serbia Nov. 20-22, , 2001, 1-5.

[13] Ravindra K., Sarma A.D., Prasad M. V. S. N., An Adaptive Polynomial Path Loss Model at UHF frequencies for Mobile railway Communications. Indian Journal of Radio and Space Physics, 31, 278-284.

[14] Huber P.J., Ronchetti E. M. Robust Statistics", second edition John Wiley \& Sons, Inc., publication, 2009.

[15] Schumacker R.E., Monahan M.P., Mount R. E., A comparison of OLS and robust regression using S-PLUS. Multiple Linear Regression Viewpoints, 28(2), 10-13, 2002.

[16] Ericsson Radio Systems AB, TEMS Cell Planner 3.4 User Guide, 2001. 
[17] Milanovic J., Rimac-Drlje. S. Bejuk K. Comparison of propagation model accuracy for WiMAX on 3.5GHz. Proceedings of the 14th IEEE, International Conference on Electronic Circuits and Systems, Dec. 11-14, 2007. IEEE Xplore Press, Morocco, 111-114. DOI: 10.1109/ICECS.2007.4510943

[18] Fratu O., Martian A., Craciunescu R., Vulpe A., Halunga S., Zaharis Z., Lazaridis P., Kasampalis S. Comparative study of Radio Mobile and ICS Telecom propagation prediction models for DVB-T. In IEEE BMSB 2015 International Conference, 17th - 19th June 2015, Ghent, Belgium.

[19] Isabona J., Isaiah G.P. Computation and Verification of Propagation Loss Models based on Electric Field Data in Mobile Cellular Networks, Australian Journal of Basic and Applied Sciences, 9(31), 280-285, 2015 .

[20] Isabona, J. Wavelet Generalized Regression Neural Network Approach for Robust Field Strength Prediction, Wireless Personal Communication (Springer) https://doi.org/10.1007/s11277-020-07550-5, 2020.

\section{Authors' Profiles}

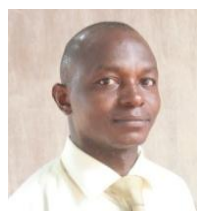

Joseph Isabona, Ph.D, received Ph.D. and M.Sc. degrees in Communication Electronics, 2013 and 2007 respectively, and a B.Sc in Applied Physics in 2003. He is the author of more than 100 scientific contributions including articles in international refereed Journals and Conferences in the area of Wireless Mobile communications. The Author is a Postdoctoral Research Fellow of the Department of Electronic Engineering, Howard College, University of KwaZulu-Natal, Durban, South Africa. His area of interest includes Signal with josabone@yahoo.com.

Processing, Radio Resource Management and Physics of radio signal propagation engineering. She can be reached

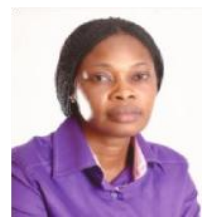

Dr. (Mrs.) O. D. Ojuh is an academic staff of Benson Idahosa University, Benin City, where she lectures Physics. She obtained her Ph.D. and M.Sc in Theoretical/ Mathematical Physics at the University of Benin City, Nigeria in 2012 and 2007 respectively and a B.Sc in Physics in 1996, from then Edo State University now known as Ambrose Alli University, Ekpoma, Edo State. Her research interest are Computational condensed matter physics/materials Science for renewable energy applications and Physics of radio signal propagation engineering. She can be reached through Email dojuh@biu.edu.ng.

How to cite this paper: Isabona Joseph, Divine O. Ojuh, " Adaptation of Propagation Model Parameters toward Efficient Cellular Network Planning using Robust LAD Algorithm", International Journal of Wireless and Microwave Technologies(IJWMT), Vol.10, No.5, pp. 13-24, 2020.DOI: 10.5815/ijwmt.2020.05.02 